\title{
Pseudomonas syringae pv. tomato DC3000 Improves Escherichia coli O157:H7 Survival in Tomato Plants
}

\author{
Min Namgung ${ }^{1 \dagger}$, Yeon-Jeong Lim ${ }^{1}$, Min Kyu Kang ${ }^{1}$, Chang-Sik $\mathrm{Oh}^{2}$, and Duck Hwan Park ${ }^{1 *}$ \\ ${ }^{1}$ Applied Biology Program, Division of Bioresource Sciences, Kangwon National University, Chuncheon 24341, Republic of Korea \\ ${ }^{2}$ Department of Horticultural Biotechnology, Kyung Hee University, Yongin 17104, Republic of Korea
}

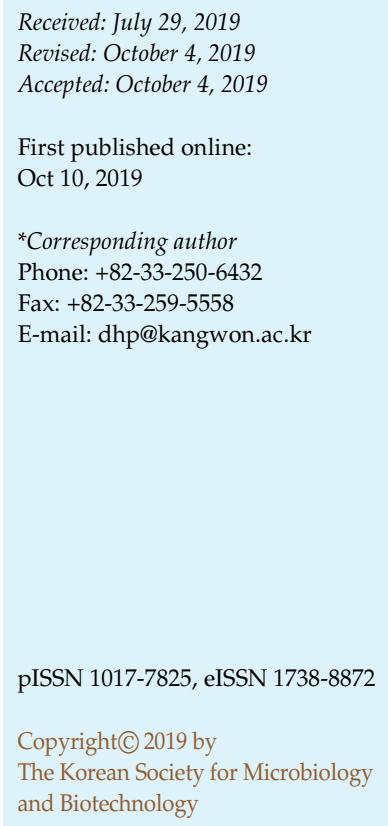

Recently, outbreaks of food-borne diseases linked to fresh produce have been an emerging public health concern worldwide. Previous research has shown that when human pathogens co-exist with plant pathogens, they have improved growth and survival rates. In this study, we have assessed whether Escherichia coli O157:H7 benefits from the existence of a phytopathogenic bacterium and the underlying mechanisms were further investigated. When Pseudomonas syringae pv. tomato DC3000 (Pst DC3000) and E. coli O157:H7 were co-inoculated by either dipping or infiltration methods, the populations of E. coli O157:H7 increased; however, no effect was observed when type three secretion system (T3SS) mutants were used instead, suggesting that E. coli O157:H7 benefits from the presence of Pst DC3000. In addition, this study confirmed that the E. coli O157:H7 populations increased when they occupied the tomato leaf intercellular space; this colonization of the interior of the leaves was possible due to the suppression of the PAMP-triggered immunity (PTI) by Pst DC3000, in particular with the AvrPto effector. In conclusion, our data support a plausible model that E. coli O157:H7 benefits from the presence of Pst DC3000 via AvrPto suppression of the PTI resistance.

Keywords: AvrPto, E. coli O157:H7, effector, food-borne disease, Pseudomonas syringae pv. tomato DC3000, tomato

\section{Introduction}

In 1996, Escherichia coli O157:H7 infected more than 10,000 Japanese children who had eaten contaminated radish sprouts [1, 2]. In addition, spinach, lettuce, and tomato have been sources of major outbreaks of E. coli O157:H7 and Salmonella, in the United States [3]. These outbreak reports demonstrate that fresh produce can be a vehicle for pathogen transfer to humans. Thus, fresh produce contaminated with human pathogens has subsequently become a topical issue around the world. Plants may be contaminated with human pathogens during the preharvest as well as the post-harvest handling stages. In the pre-harvest stage, irrigation water from manure or animal feces are the major causes of contamination, whereas nonsanitary processing during transportation and bagging are the major sources of contamination in the post-harvest stage $[1,4]$. However, the focus of previous research has been on the risks of food-borne illness and contamination routes rather than the actual relationships between the human and plant pathogens.

Past studies found that when food-borne pathogens internally contaminate a plant, they can survive longer than when they are only on plant surfaces $[4,5]$. Furthermore, food-borne pathogens are recognized by the plant immune responses $[6,7]$, and in cases where they co-exist with plant pathogens, they benefit from the behaviors of these same pathogens $[8,9]$. There are relatively few reports that deal with the relationship between human and plant pathogens. Greater understanding of the biological interactions between humans and plant pathogens is indispensable to prevent outbreaks of human pathogens. In the present study, the internalization of E. coli O157:H7 in tomato plants, the enhancement of plant immunity, and the mechanisms from which E. coli O157:H7 benefits in the presence of a plant pathogenic bacterium have been investigated. 


\section{Materials and Methods}

\section{Bacterial Strains and Growth Conditions}

Bacterial strains used in the current study are described in Table 1. The E. coli O157:H7 strain ATCC 43895 and a GFP-tagged version were grown on Luria-Bertani (LB) medium at $37^{\circ} \mathrm{C}$ for 16 h. For the count of E. coli O157:H7 from the inoculated plants, Eosin Methylene Blue (EMB) medium (Difco, USA) was used. P. syringae pv. tomato DC3000 (Pst DC3000) and its derivatives, and P. fluorescens (Pf) were cultured in King's B (KB) at $28^{\circ} \mathrm{C}$ for 1 day. Antibiotics were added to the culture medium at the following concentrations: $50 \mu \mathrm{g} / \mathrm{ml}$ kanamycin $(\mathrm{Km})$ for the E. coli O157:H7 GFP tagging strain; $20 \mu \mathrm{g} / \mathrm{ml}$ chloramphenicol $(\mathrm{Cm})$ and $50 \mu \mathrm{g} / \mathrm{ml}$ ampicillin (Am) for Pf; $50 \mu \mathrm{g} / \mathrm{ml}$ rifampicin (Rif) for Pst; $50 \mu \mathrm{g} / \mathrm{ml}$ spectinomycin (Sp) for its derivative mutants.

\section{Bacterial Inoculations and Population Tests}

Fully expanded leaves from 5-week-old tomato (Solanum lycopersicum 'Prime Alexander') were used for the analysis of survival rates, the population size over time, and internal colonization by the E. coli O157:H7 strain. To prepare bacterial inoculum, the E. coli O157:H7 strain was incubated on solid LB medium and the cells were then suspended in $10 \mathrm{mM} \mathrm{MgCl}_{2}$ at the concentration of $1 \times 10^{7} \mathrm{CFU} / \mathrm{ml}$ with $0.02 \%$ (v/v) Silwet L-77 surfactant (Lehle Seeds Co., USA). Two leaflets on the third branch of each tomato plant were dip-inoculated in the bacterial suspension for $2 \mathrm{~min}$. For infiltration, a bacterial suspension of $1 \times 10^{5} \mathrm{CFU} / \mathrm{ml}$ was infiltrated into tomato leaves of the third branch using blunt syringes. Inoculated plants were kept at $26^{\circ} \mathrm{C}$ for $10 \mathrm{~h}$ of illumination. Leaf disks were sampled 10, 20, and 30 days post inoculation (DPI) from both dipped and infiltrated inoculations using a $1 \mathrm{~cm}$-diameter cork borer. The samples were then ground in $1 \mathrm{ml} 10 \mathrm{mM} \mathrm{MgCl} 2$ using a plastic pestle. The samples were then diluted tenfold and $100 \mu \mathrm{l}$ aliquots were plated on $\mathrm{EMB}$ medium and grown at $37^{\circ} \mathrm{C}$ overnight, after which the bacterial colonies were measured. To investigate E. coli O157:H7 populations in plants over time, bacterial suspensions of

Table 1. Bacterial strains used in this study.

\begin{tabular}{|c|c|}
\hline Strain & Genotype or relative phenotype \\
\hline \multicolumn{2}{|l|}{ Escherichia coli } \\
\hline O157:H7 ATCC 43895 & Wild-type \\
\hline GFP labeling & pBAV1K-t5-gfp; Km ${ }^{\mathrm{R}}$ \\
\hline Pseudomonas fluorescens 55 & Wild type $\mathrm{Cm}^{\mathrm{R}}, \mathrm{Am}^{\mathrm{R}}$ \\
\hline \multicolumn{2}{|l|}{ P. syringae } \\
\hline pv. tomato DC3000 & Wild-type Rif ${ }^{\mathrm{R}}$ \\
\hline CUCPB5112 & $\Delta h r c Q_{\mathrm{b}}:: F R T ; \mathrm{Sp}^{\mathrm{R}}$ \\
\hline CUCPB5535 & $\Delta a v r P t o B ; \operatorname{Rif}^{\mathrm{R}} \mathrm{Ap}^{\mathrm{R}}$ \\
\hline CUCPB5543 & $\Delta a v r P t o B / a v r P t o ; \operatorname{Rif}^{\mathrm{R}} \mathrm{Ap}^{\mathrm{R}}$ \\
\hline CUCPB5585 & $\Delta 28$ effectors; $\operatorname{Rif}^{\mathrm{R}} S \mathrm{p}^{\mathrm{R}}$ \\
\hline
\end{tabular}

$1 \times 10^{4} \mathrm{CFU} / \mathrm{ml}$ were directly infiltrated into tomato leaves and the bacterial colonies were then counted at 1, 2, 3, 4, and 5 DPI. To determine the internalization of E. coli O157:H7, one of the two leaves from each of the dip-inoculated plants described above, was sterilized with $70 \%$ ethanol for $20 \mathrm{~s}$ and then $0.5 \%$ sodium hypochlorite for $10 \mathrm{~s}$ and then washed with sterilized distilled water thrice for $5 \mathrm{~min}$ each time. Leaf disks were punched out using a $1 \mathrm{~cm}$-diameter cork borer. The leaf disks were macerated with a plastic pestle, grown on medium and the bacterial colonies were counted as described above. The non-sterilized leaf was considered the negative control. The experiments were repeated three times with similar results.

For stomatal integration, the E. coli O157:H7 strain tagged with the green fluorescent protein (GFP) gene was grown to an $\mathrm{OD}_{600}$ of 0.6 in LB broth and incubated for $12 \mathrm{~h}$ after the addition of $0.5 \mathrm{mM}$ IPTG, then pelleted from the broth, and the cells were resuspended in $10 \mathrm{mM} \mathrm{MgCl}_{2}$ to an $\mathrm{OD}_{600}$ of 0.6 supplemented with $0.02 \%$ of Silwet L-77. Tomato leaves were dip-inoculated and then incubated for $2 \mathrm{~h}$ at room temperature, after which leaf pieces were excised and mounted with $70 \%$ glycerol in PBS buffer. GFP-labeled bacteria were visualized using a super sensitive high resolution confocal laser scanning microscope (Zeiss LSM 880, Germany). Fluorescent bacterial scanning using an excitation wavelength of $488_{\mathrm{nm}}$ and a $502-534_{\mathrm{nm}}$ emission filter were conducted.

\section{Callose Deposition}

A callose deposition assay for Nicotiana benthamiana was modified as follows from a previous report [10].

\section{Co-Inoculation of E. coli O157:H7 with P. syringae pv. tomato DC3000}

To test whether E. coli O157:H7 benefits from the co-inoculated plant pathogenic bacteria, we inoculated E. coli O157:H7 with Pst DC3000 and its derivatives that lack a type three secretion system (T3SS) and effectors. Concentrations of $1 \times 10^{5} \mathrm{CFU} / \mathrm{ml}$ for each strain were mixed with $10 \mathrm{mM} \mathrm{MgCl}$ and the mixture was infiltrated directly into the tomato leaves. At 1 DPI, disks were collected and E. coli O157:H7 was grown on an EMB plate as mentioned above. Besides the leaves, $1.0-1.5 \mathrm{~cm}$ immature fruits of tomato were also used for co-existence analysis. The immature fruits were cut into two halves and the round surfaces placed on sterilized glass slides above filter paper in glass petridishes, and $20 \mu \mathrm{l}$ of mixture with both E. coli O157:H7 and Pst DC3000 at $1 \times$ $10^{5} \mathrm{CFU} / \mathrm{ml}$ concentrations were dropped onto the upper and sliced surfaces. Immature fruits $(0.1 \mathrm{~g})$ were sectioned and used to assess bacterium populations using the same method described above.

The experiments were performed in three replications with 20 leaves in the leaf assay and 18 immature fruits in the fruit assay per replication. The experiments were repeated at least three times. Statistical significance was considered by analysis of variance (ANOVA) and Duncan's multiple range test for $p$ values $=0.05$. 


\section{Results}

\section{E. coli O157:H7 Can Colonize in Tomato Leaves}

Previous reports [11, 12] confirmed that E. coli O157:H7 can colonize and survive on the plant surface and in the interior of plants, including tomato skins, spinach, and lettuce leaves. We analyzed how long E. coli O157:H7 can survive in tomato leaves using both dip and infiltrationcontamination methods. The study showed that E. coli O157:H7 populations were maintained for 30 days reaching 4.75 and $3.92 \log$ CFU/disk, for each method respectively, which were similar to the initial concentrations from the dip and infiltration inoculation methods (Fig. 1A). These results signify that E. coli $\mathrm{O} 157: \mathrm{H7}$ is able to inhabit and survive for more than one month in a plant. Therefore, the infiltration inoculation was deemed suitable for further experiments to observe the populations of E. coli O157:H7 in plants. We infiltrated leaves with bacteria to analyze the population increases over time. As shown in Fig. 1B, the population increased at $1 \mathrm{DPI}$ and the level achieved was maintained until 5 DPI, thus, 1 DPI was sufficient to have confidence of colonization of E. coli O157:H7 in the interior of leaves. In order to examine the internalization of $E$. coli O157:H7, we prepared non-disinfected and disinfected samples using ethanol and sodium hypochlorite. The populations of E. coli $\mathrm{O} 157: \mathrm{H7}$ on the non-disinfected
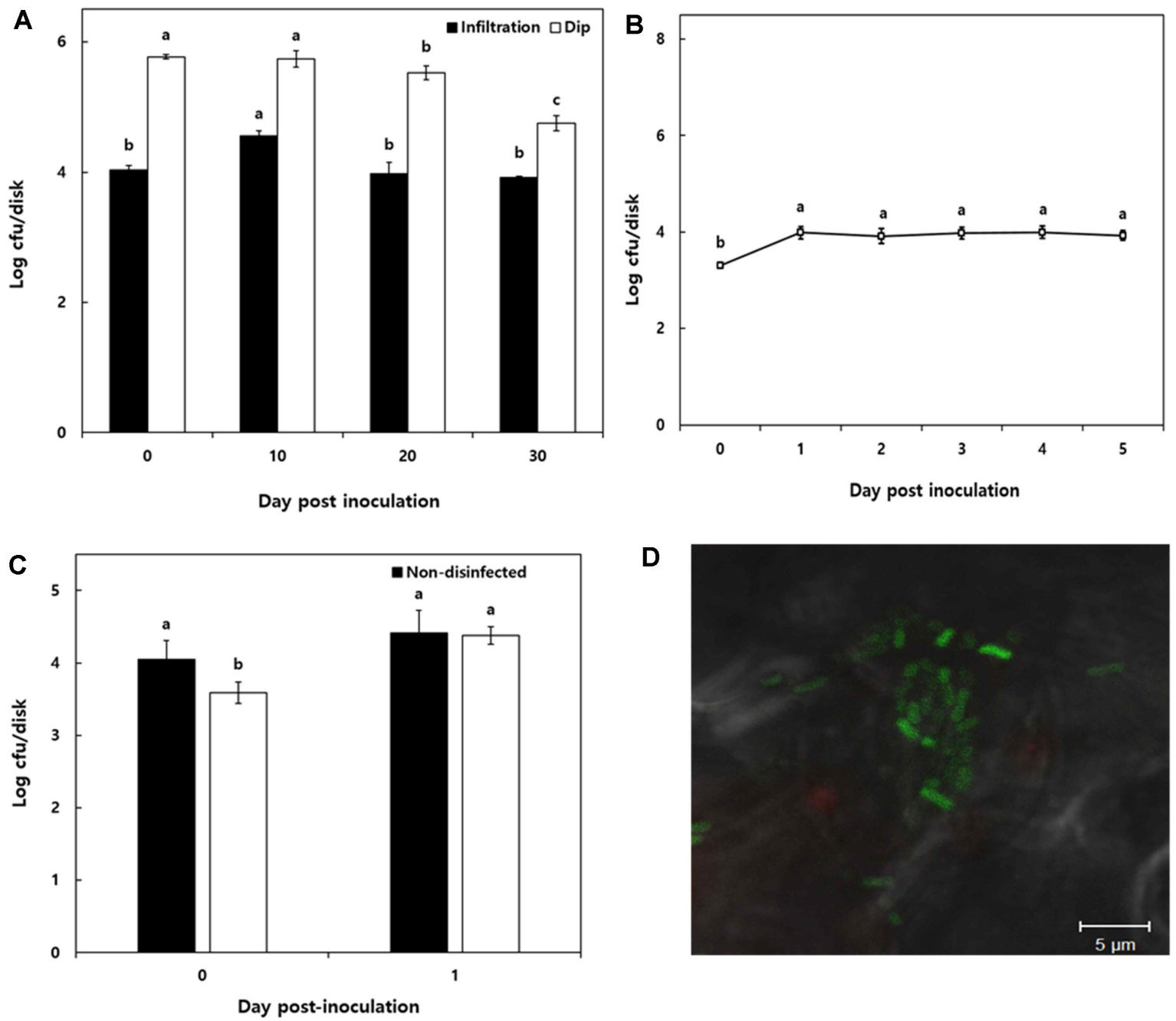

D

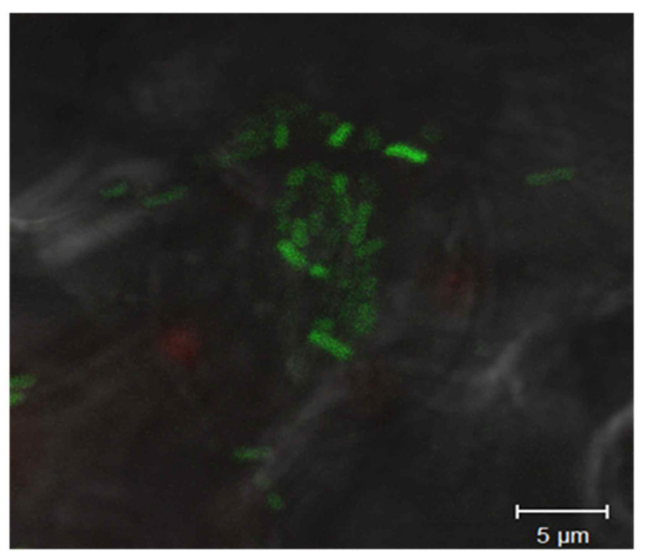

Fig. 1. Populations of E. coli O157:H7 in tomato (Solanum lycopersicum 'Prime Alexander') leaves.

(A) Survival ratio of E. coli O157:H7 based on the time course until 30 days post inoculation for the dip and infiltrated-inoculations. (B) Increasing time points and the population of E. coli O157:H7 by dip-inoculation. (C) Populations of E. coli O157:H7 on the surface and in the interior of tomato leaves with and without surface disinfection. (D) Confocal image of GFP-tagged E. coli O157:H7 cells colonizing a tomato leaf with numerous proliferated cells around a stomate. 
leaves were $4.05 \log \mathrm{CFU} /$ disk compared to the disinfected leaves at 3.59 CFU/disk (Fig. 1C). After 1 day, however, the populations on the disinfected leaves were found to be similar to the non-disinfected ones, suggesting that E. coli O157:H7 had colonized the interior of the tomato leaves. In addition, the GFP-labeled E. coli O157:H7 strain was observed near and seemed to be associated with the leaf stomata (Fig. 1D). The internal colonization of E. coli O157:H7 in the tomato leaves was subsequently thought to have entered through the stomata.

\section{E. coli 0157:H7 Induces the Immune Response in Plant}

To test whether E. coli O157:H7 elicits immune responses in plants, we used callose deposition assays to assess for PAMP-Triggered Immunity (PTI) in leaves of N. benthamiana. The $P$. fluorescence 55 strain induced numerous calloses in $N$. benthamiana, but $10 \mathrm{mM} \mathrm{MgCl}$ did not as indicated in Fig. 2A. E. coli O157:H7 induced a similar number of calloses to that of $P$. fluorescence (Fig. 2B), which was the representative strain used to induce callose deposition for PTI responses in plants [10].
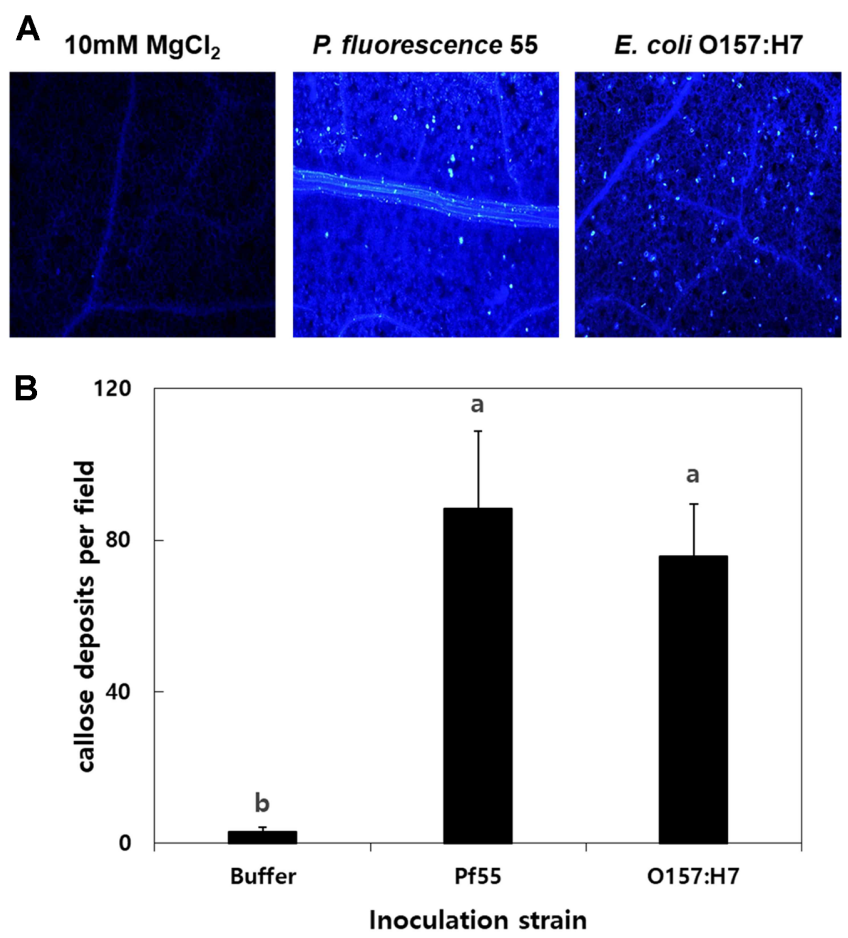

Fig. 2. Callose deposition in Nicotiana benthamiana.

Leaves were syringe-infiltrated with Pseudomonas fluorescence 55, E. coli O157:H7, or $10 \mathrm{mM} \mathrm{MgCl}_{2}$ buffer and assayed at the indicated times using aniline blue and fluorescence microscopy. A, Representative callose deposits of $1 \mathrm{~mm}^{2}$ in field were captured using a Leica DMI 3000B. B, The mean number of callose deposits from three replicates.
P. syringae pv. tomato DC3000 Enhances the Ability of E. coli $\mathrm{O} 157: \mathrm{H} 7$ to Colonize in Tomato

Since E. coli O157:H7 was able to colonize the interior of tomato plants, we tested whether E. coli O157:H7 may benefit from the co-inoculated plant pathogenic bacteria. When E. coli O157:H7 was co-inoculated in leaves with mutants of Pst DC3000, which lacked the T3SS and 28 effectors, respectively, E. coli O157:H7 populations were not increased at 1 DPI (Fig. 3A). Whereas, E. coli O157:H7 co-inoculated with Pst DC3000 had significantly increased
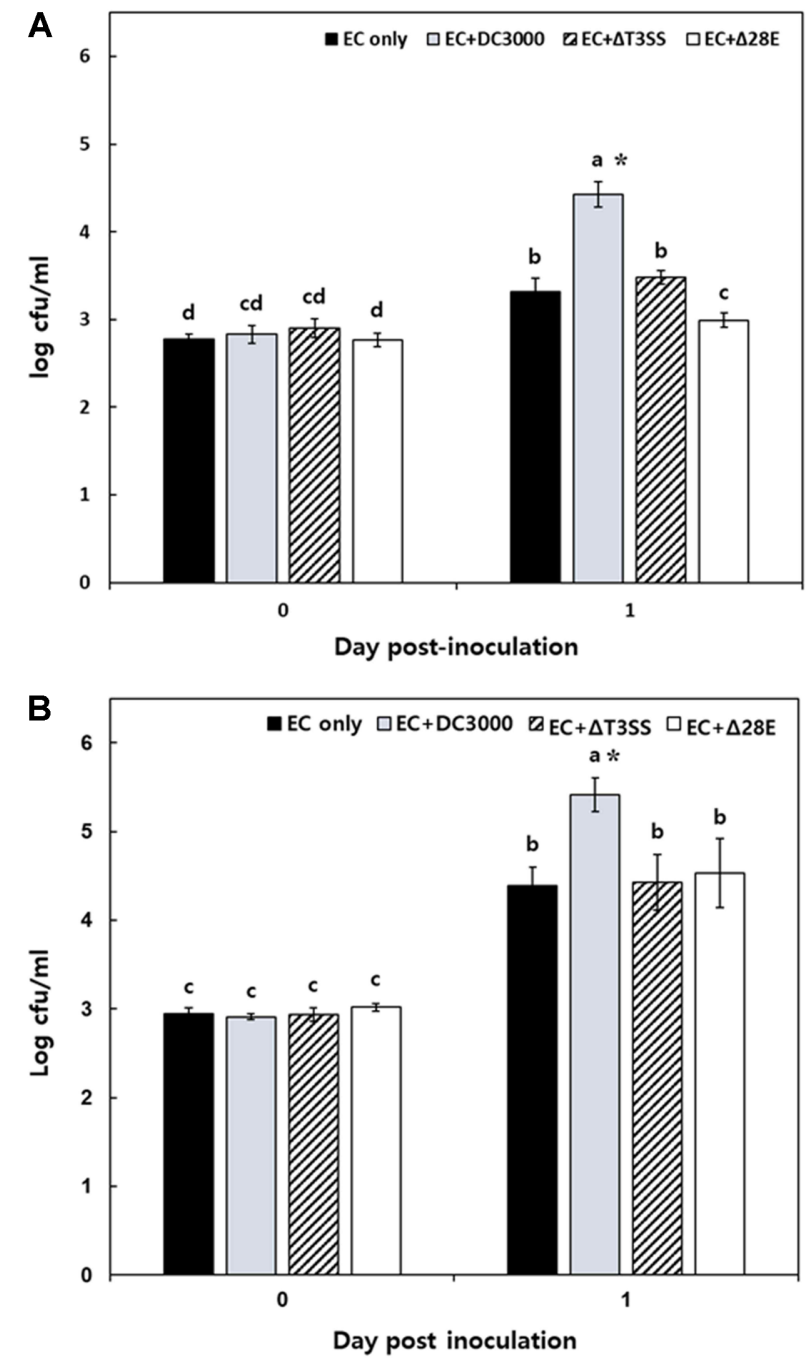

Fig. 3. Population dynamics of E. coli O157:H7 inoculated with Pst DC3000 and its mutants on tomato leaves (A) and fruits $(\mathbf{B})$.

Results shown are the mean number of counted E. coli O157:H7 cells from three replicates and the standard deviation from 20 leaves from three samples for each treatment \pm standard error. Significant differences in populations compared with E. coli O157:H7 are indicated with an asterisk $(p=0.05)$. 
populations (Fig. 3A). These findings were similar to those when the two bacteria were inoculated in immature fruits of tomato. Moreover, the recovered populations of E. coli O157:H7 from immature fruits were higher than the population in leaves. These results suggest that the survival ability of E. coli O157:H7 is enhanced when associated with the plant pathogenic bacterium, Pst DC3000. The plausible explanation is likely due to the suppression of the plant immune system evoked by E. coli O157:H7 and Pst DC3000. Additionally, E. coli O157:H7 may be more easily adopted into fruits than leaves, suggesting that contact with contaminated sources during the pre-harvest season must be avoided for food safety.

\section{E. coli 0157:H7 Can Propagate in Plants by Avoiding PTI} Via the avrPto of $P$. syringae pv. tomato DC3000

In this study, Pst DC3000 had a key role in enhancing the capability of E. coli O157:H7 to survive in tomato plants via avoiding the plant immunity response. However, it was still unclear which mechanism was helping E. coli O157:H7 to survive in the plant. The mutants of T3SS and those with the 28 effector deletions did not result in increased populations of E. coli O157:H7 and it was thought that the roles of the deleted effectors may be to suppress plant immunity. To examine this hypothesis, we used the effectors AvrPto and AvrPtoB, which are known to suppress PTI. When E. coli O157:H7 was co-inoculated with the wild type of Pst DC3000 and $\triangle a v r P t o B$ in tomato leaves and fruits, E. coli O157:H7 populations were significantly increased but not with $\Delta 28$ effectors and $\triangle a v r P t o B / a v r P t o$ double mutants (Figs. 4A and 4B). Thus, we concluded that E. coli O157:H7 benefitted from AvrPto suppression of PTI.

\section{Discussion}

In this study, survival periods of E. coli O157:H7 were estimated for 30 DPI for both the dip and infiltrationinoculation methods. It was previously found that human pathogenic bacteria, particularly E. coli O157:H7 and S. enterica serovar Typhimurium, were able to survive for 30 and 25 days when inoculated on lettuce and tomato plants using dip and spray inoculation methods, respectively [5, 13]. Thus, the survival rate of 30 days in tomato leaves was considered reasonable in our inoculation regime. The ability of E. coli O157:H7 to enter into the tomato plant via the stomata and colonize it internally was confirmed by confocal observations and disinfection analysis with $70 \%$ ethanol and $0.5 \%$ hypochlorite, in agreement with previous studies on entero-aggregative E. coli [11] and S. enterica [5].
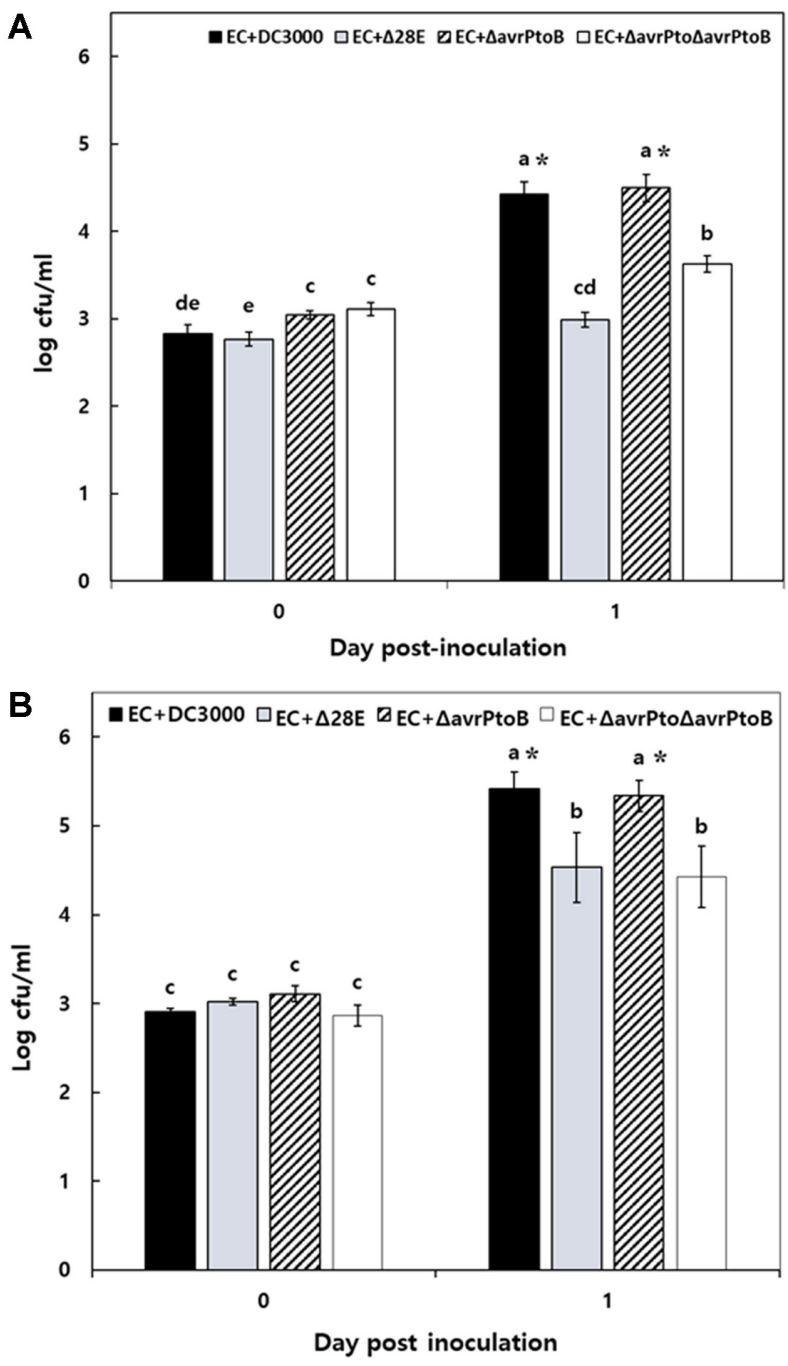

Fig. 4. Population dynamics of E. coli O157:H7 inoculated with Pst DC3000 and its AvrPtoB and AvrPtoB/AvrPto double mutants on tomato leaves (A) and fruits (B).

Results shown are the mean number of counted E. coli O157:H7 cells from three replicates and standard deviations from 18 fruits from three samples for each treatment, \pm standard error. Significant differences in populations between the E. coli O157:H7 and mutants of Pst DC3000 are indicated with asterisks $(p=0.05)$.

Therefore, we concluded that the stomata may be the critical site for E. coli O157:H7 to enter the tomato plant and further grow in the apoplast space. This allows the bacteria to avoid external stresses such as UV irradiation and desiccation; this behavior is similar to that of S. enterica [14] whose population was maintained in this manner for more than one month, which was enough to lead to contamination of a subsequent host.

In earlier studies, E. coli O157:H7 did not induce plant 
immunity [15]. However, on Arabidopsis, the population of the E. coli O157:H7 flagella mutant strain was higher than the wild type population, indicating that the flagella of the E. coli O157:H7 wild type were recognized by Arabidopsis [7]. In our study, E. coli O157:H7 induced numerous callose depositions, on a level similar to those induced by $P$. fluorescence 55. Callose depositions were considered to indicate cell wall-based defense activation following the perception of PAMPs (Pathogen-Associated Molecular Patterns) including flagellin, elongation factor $\mathrm{Tu}$, lipopolysaccharides, and glycoproteins as a preliminary defense of the PTI in plants $[16,17]$. The population of E. coli $\mathrm{O} 157: \mathrm{H7}$ on dip-inoculated leaves declined gradually over time, which may be attributed to plant responses triggered by perceptions of PAMPs, in particular with flagella of E. coli O157:H7 (Fig. 1A). However, we could not detect stomatal closure to prevent bacteria entering the plant due to E. coli O157:H7 exposure, as has been previously reported [6]. The population tests and callose depositions observed in the current study supported the idea that E. coli $\mathrm{O} 157: \mathrm{H7}$ is capable of entering the plant epidermis via the stomata and growing endophytically, and PTI is triggered in the process of penetration by bacteria like Salmonella [8]. However, E. coli O157:H7 that multiplied or was sustained in the tomato leaves did not cause any symptoms after its populations were detected at concentrations of at least $\log 4$ and $6 \mathrm{CFU} / \mathrm{ml}$ in the case of infiltrated and dip-inoculated leaves, suggesting that $E$. coli O157:H7 triggers PTI, not ETI (effector-triggered immunity) in agreement with previous reports that human pathogens did not have T3SS (type III secretion system) to inject effectors into plant cells [4].

When PTI was triggered by E. coli $\mathrm{O} 157: \mathrm{H7}$, the population of E. coli O157:H7 in the tomato declined slightly over time, whereas E. coli O157:H7 populations were significantly increased when they were co-inoculated with Pst DC3000. Similarly, previous studies reported the key role of phytopathogens for the survival of human pathogens. Meng and colleagues [8] reported that Pst DC3000 was able to significantly enhance the growth of Salmonella in N. benthamiana and also co-inoculations with Xanthomonas perforans influenced Salmonella colonization in tomato phyllospere [18]. Furthermore, populations of E.coli O157:H7 and Salmonella were increased in necrotic lesions on leaves of romaine lettuce caused by the downy mildew pathogen, Bremia lactucae [9]. In addition, the finding that populations of E. coli O157:H7 were not increased by coinoculation with $\triangle \mathrm{T} 3 S S$ and $\triangle 28 \mathrm{E}$ mutants supports the hypothesis that human pathogens may benefit from association with plant pathogenic bacteria via the suppression of immune responses by the T3SS effectors, which were delivered into plant cells by plant pathogens [8].

AvrPto and AvrPtoB effectors were chosen for this study because of their modes of action to circumvent plant defenses [19-22]. Two effector proteins can share the same host targets and also manipulate multiple host factors [23]. For example, AvrPto suppresses the kinase activity of FLS2 and EFR, and AvrPtoB that includes an E3 ubiquitin ligase domain can inhibit many PRRs such as FLS2, FEN, CERK1, and Bti9 [24]. The results showed that the growth of E. coli O157:H7 co-inoculated with $\triangle a v r P t o B$ was similarly increased to that of the wild type but not $\triangle a v r P t o B / a v r P t o$, suggesting that the mechanism of AvrPto is important for E. coli O157:H7 to colonize plants. Although we did not perform a complementation test for these effectors in the preset study, AvrPto helps survival of E. coli O157:H7 in tomato plant; therefore, AvrPto and AvrPtoB must be complementary to those deletion mutants and the 28 effectors deletion mutant in future study. Additionally, the growth of E. coli O157:H7 in fruits was higher than in leaves. The $\mathrm{pH}$ of immature and mature fruits is 6.7 and 4.4 respectively [25] but, does not seem to affect the growth of E. coli O157:H7 as it was found to be able to survive in epidermal fruit tissues in which the $\mathrm{pH}$ was neutral. This highlights the contamination risks present with tomato fruits. Therefore, food-borne illness outbreaks are possible if raw fresh produce contaminated with human pathogens is consumed.

Based on our data, E. coli O157:H7 can induce PTI, an early immune response in plants, in the process of penetration and colonization of the plant and can propagate by avoiding the PTI with the assistance of Pst DC3000, via its effector, AvrPto. Thus, a better understanding of the relationships between plant and human pathogens will help to develop effective strategies in reducing food-borne illnesses and maintaining human health.

\section{Acknowledgements}

This research was supported by the Basic Science Research Program through the National Research Foundation of Korea (NRF) funded by the Ministry of Education (No. 2018R1A6A1A03025582).

\section{Conflict of Interest}

The authors have no financial conflicts of interest to declare. 


\section{References}

1. Barak JD, Schroeder BK. 2012. Interrelationships of food safety and plant pathology: the life cycle of human pathogens on plants. Annu. Rev. Phytopathol. 50: 241-266.

2. Watanabe Y, Ozasa K, Mermin JH, Griffin PM, Masuda K, Imashuku S, et al. 1999. Factory outbreak of Escherichia coli O157:H7 infection in Japan. Emerg. Infect. Dis. 5: 424-428.

3. Doyle MP, Erickson MC. 2008. Summer meeting 2007 - the problems with fresh produce: an overview. J. Appl. Microbiol. 105: 317-330.

4. Melotto M, Panchal S, Roy D. 2014. Plant innate immunity against human bacterial pathogens. Front Microbiol. 5: doi: 10.3389/fmicb.2014.00411.

5. Gu G, Hu J, Cevallos-Cevallos JM, Richardson SM, Bartz JA, van Bruggen AHC. 2011. Internal colonization of Salmonella enterica serovar Typhimurium in tomato plants. PLoS One 6: $\mathrm{e} 27340$.

6. Roy D, Ranchal W, Rosa BA, Melotto M. 2013. Escherichia coli O157:H7 induces plant immunity than Salmonella enterica Typhimurium SL1344. Phyhtopathology 103: 326-332.

7. Seo S, Matthews KR. 2012. Influence of the plant defense response to Escherichia coli O157:H7 cell surface structures on survival of that enteric pathogen on plant surfaces. Appl. Environ. Microbiol. 78: 5882-5889.

8. Meng F, Altier C, Martin GB. 2014. Salmonella colonization activates the plant immune system and benefits from association with plant pathogenic bacteria. Environ. Microbiol. 15: $2418-2430$.

9. Simko I, Zhou Y, Brandl M. 2015. Downy mildew disease promotes the colonization of romain lettuce by Escherichia coli O157:H7 and Salmonella enterica. BMC Microbiol. 15: 19. doi: 10.1186/s12866-015-0360-5.

10. Nguyen HP, Chakravarthy S, Velásquez AC, McLane HL, Zeng L, Nakayashiki $\mathrm{H}$, et al. 2010. Methods to study PAMP-triggered immunity using tomato and Nicotiana benthamiana. Mol. Plant Microbe Interact. 23: 991-999.

11. Berger CN, Sodha SV, Shaw RK, Griffin PM, Pink D, Hand P, et al. 2010. Fresh fruit and vegetables as vehicles for the transmission of human pathogens. Environ. Microbiol. 12: 2385-2397.

12. Wright KM, Chapman S, McGeachy K, Humphris S, Campbell E, Toth IK, et al. 2013. The endophytic lifestyle of Escherichia coli O157:H7: quantification and internal localization in roots. Phytopathology 103: 333-340.

13. Solomon EB, Pang HJ, Matthews KR. 2003. Persistence of Escherichia coli O157:H7 on lettuce plants following spray irrigation with contaminated water. J. Food Prot. 66: 21982202.
14. Kroupitski Y, Golberg D, Belausov E, Pinto R, Swartzberg $\mathrm{D}$, Granot D, et al. 2009. Internalization of Salmonella enterica in leaves is induced by light and involves chemotaxis and penetration through open stomata. Appl. Environ. Microbiol. 75: 6076-6086.

15. Thilmony R, Underwood W, He SY. 2006. Genome-wide transcriptional analysis of the Arabidopsis thaliana interaction with the plant pathogen Pseudomonas syringae pv. tomato DC3000 and the human pathogen Escherichia coli O157:H7. Plant J. 46: 34-53.

16. Hauck P, Thilmony R, He SY. 2003. A Pseudomonas syringae type III effector suppresses cell wall-based extracellular defense in susceptible Arabidopsis plants. Proc. Natl. Acad. Sci. USA 100: 8577-8582.

17. Nürnberger T, Brunner F, Kemmerling B, Piater L. 2004. Innate immunity in plants and animals: striking similarities and obvious differences. Immunol. Rev. 198: 249-266.

18. Potnis N, SotoArias JP, Cowles KN, van Bruggen AH, Jones JB, Barak JD. 2014. Xanthomonas perforans colonization influences Salmonella enterica in the tomato phyllosphere. Appl. Environ. Microbiol. 80: 3173-3180.

19. Abramovitch RB, Kim Y-J, Chen S, Dickman MB, Martin GB. 2003. Pseudomonas type III effector AvrPtoB induces plant disease susceptibility by inhibition of host programmed cell death. EMBO J. 22: 60-69.

20. Chang JH, Rathjen JP, Bernal AJ, Staskawicz BJ, Michelmore RW. 2000. AvrPto enhances growth and necrosis caused by Pseudomonas syringae pv. tomato in tomato lines lacking either Pto or Prf. Mol. Plant Microbe Interact. 13: 568-571.

21. Shan L, Thara VK, Martin GB, Zhou JM, Tang X. 2000. The Pseudomonas AvrPto protein is differentially recognized by tomato and tobacco and is localized to the plant plasma membrane. Plant Cell 12: 2323-2338.

22. Wei H-L, Chakravarthy S, Mathieu J, Helmann TC, Stodghill P, Swingle B, et al. 2015. Pseudomonas syringae pv. tomato DC3000 type III secretion effector polymutants reveal an interplay between HopAD1 and AvrPtoB. Cell Host Microbe 17: 752-762.

23. Shan L, He P, Li J, Heese A, Peck SC, Nürnberger T, et al. 2008. Bacterial effectors target the common signaling partner BAK1 to disrupt multiple MAMP receptor-signaling complexes and impede plant immunity. Cell Host Microbe 4: 17-27.

24. Xin X, He SY. 2013. Pseudomonas syringae pv. tomato DC3000: a model pathogen for probing disease susceptibility and hormone signaling in plants. Annu. Rev. Phytopathol. 51: 473498.

25. Almeida DP, Huber DJ. 1999. Apoplastic $\mathrm{pH}$ and inorganic ion levels in tomato fruit: a potential means for regulation of cell wall metabolism during ripening. Physiol. Plantarum 105: 506-512. 